\title{
Evaluation of acute bronchodilator reversibility in patients with symptoms of GOLD stage I COPD
}

\author{
D E O'Donnell, ${ }^{1}$ P Laveneziana, ${ }^{1}$ J Ora, ${ }^{1}$ K A Webb, ${ }^{1}$ Y-M Lam, ${ }^{2}$ D Ofir ${ }^{1}$
}

\begin{abstract}
- Additional Methods data are published online only at http:// thorax.bmj.com/content/vol64/ issue3

${ }^{1}$ Respiratory Investigation Unit, Department of Medicine,

Queen's University and Kingston General Hospital, Kingston, Ontario, Canada; ${ }^{2}$ Department of Community Health and Epidemiology, Queen's University, Kingston, Ontario, Canada
\end{abstract}

Correspondence to: Dr D O'Donnell, 102 Stuart Street, Kingston, Ontario, Canada K7L 2V6; odonnell@ queensu.ca

Presented in part at the ALA/ ATS International Conference, Toronto, May 2008 (Ofir D, Laveneziana $\mathrm{P}$, Webb KA, et al. Evaluation of bronchodilator efficacy in symptomatic patients with GOLD stage I COPD. Am J Respir Crit Care Med 2008:177(Suppl):A649).

Received 27 June 2008 Accepted 2 November 2008

Published Online First

26 November 2008

\section{ABSTRACT}

Background: Patients with symptoms of GOLD stage I chronic obstructive pulmonary disease (COPD) can have significant abnormalities of ventilatory mechanics with greater exertional symptoms and exercise limitation than age-matched healthy subjects. In such patients the impact of bronchodilator therapy remains unknown and is difficult to evaluate.

Methods: The acute effects of nebulised ipratropium bromide $500 \mu \mathrm{g}$ (IB) on resting pulmonary function and on dyspnoea and ventilatory parameters during symptomlimited constant work rate cycle exercise were measured. In a randomised double-blind crossover study, 16 patients with COPD (mean (SD) post-bronchodilator forced expiratory volume in $1 \mathrm{~s}\left(\mathrm{FEV}_{1}\right) \mathrm{g0}(7) \%$ predicted, $\mathrm{FEV}_{1} /$ forced vital capacity (FVC) 59 (7)\%) with a significant smoking history (mean (SD) 44 (16) pack-years) inhaled either IB or placebo on each of two separate visits. Pulmonary function tests and cycle exercise at $80-85 \%$ of each subject's maximal work capacity were performed $2 \mathrm{~h}$ after dosing.

Results: Compared with placebo, $\mathrm{FEV}_{1}$ increased 5 (9)\% predicted, residual volume decreased $12(20) \%$ predicted and specific airway resistance decreased 81 (93)\% predicted (all $\mathrm{p}<0.05$ ) after IB. At a standardised time during exercise, dynamic inspiratory capacity and tidal volume significantly increased in tandem by 0.12 and 0.16 litres, respectively (each $p<0.05$ ), dyspnoea fell by 0.9 (1.8) Borg units $(p=0.07)$ and dyspnoea/ventilation ratios fell significantly $(p<0.05)$. The fall in dyspnoea intensity at higher submaximal ventilations correlated with the concurrent decrease in end-expiratory lung volume $(p<0.05)$.

Conclusion: In patients with symptoms of GOLD stage I COPD, IB treatment is associated with modest but consistent improvements in airway function, operating lung volumes and dyspnoea intensity during exercise. These results provide a physiological rationale for a trial of bronchodilator therapy in selected patients with milder but symptomatic COPD.

Patients with chronic obstructive pulmonary disease (COPD) who have relatively preserved measurements of forced expiratory flow rates may have extensive small airway dysfunction. ${ }^{1-4}$ Such patients report greater intensity of exertional dyspnoea than healthy age-matched controls as a result of the combined effects of abnormal dynamic ventilatory mechanics and higher ventilatory requirements during exercise. ${ }^{5}$ This physiological impairment of the respiratory system may explain, at least in part, reports of poor perceived health status in subpopulations of patients with apparently mild airway obstruction. ${ }^{6}$ Successful smoking cessation is the only proven intervention that has been shown to improve small airway function in patients with mild COPD. ${ }^{24}$ However, the optimal clinical management of these smokers with symptoms of mild COPD is not established and remains largely unstudied. It is not known, for example, whether inhaled bronchodilator therapy, which has established efficacy in moderate to severe COPD,,$^{7-10}$ is effective in alleviating activity-related dyspnoea in those with milder disease. Moreover, it remains uncertain whether traditional spirometric criteria for bronchodilator reversibility, based on arbitrary improvement in the forced expiratory volume in $1 \mathrm{~s}\left(\mathrm{FEV}_{1}\right)$, are applicable in mild COPD. This information becomes important for clinical practice and for the design of future clinical trials to evaluate the efficacy of therapeutic interventions in early COPD.

The purpose of the present study was therefore to evaluate the acute effects of an anticholinergic bronchodilator on airway function and exertional dyspnoea in patients with mild COPD, as defined by GOLD stage I criteria. ${ }^{10}$ Based on the results of a previous mechanistic study in patients with symptoms of mild COPD, ${ }^{5}$ we hypothesised that inhaled bronchodilator therapy would improve airway function and lung volumes at rest and reduce the rate of dynamic pulmonary hyperinflation during exercise, thus permitting greater tidal volume expansion and reduced dyspnoea intensity at higher submaximal ventilations. To test this hypothesis we undertook a randomised placebo controlled study in 16 well characterised patients with mild COPD symptoms. We compared the acute effects of nebulised ipratropium bromide and placebo on detailed resting pulmonary function measurements as well as dyspnoea ratings, operating lung volumes, breathing pattern and gas exchange during constant work cycle exercise. To explore potential mechanisms of dyspnoea relief, we also measured oesophageal pressure (Pes)derived indices of dynamic ventilatory mechanics in a small subsample of patients who consented to undertake these more invasive measurements.

\section{METHODS}

\section{Subjects}

Sixteen patients with symptoms of GOLD stage I COPD (post-bronchodilator $\mathrm{FEV}_{1} \geqslant 80 \%$ predicted and $\mathrm{FEV}_{1} /$ forced vital capacity $(\mathrm{FVC})$ ratio $\left.<0.7\right)^{10}$ who were referred to the COPD Centre at our institution were studied. Patients were excluded if they had (1) other medical conditions which could cause or contribute to breathlessness (ie, metabolic, cardiovascular, asthma or other respiratory diseases) or (2) other disorders which could interfere 
with exercise testing such as neuromuscular diseases or musculoskeletal problems.

\section{Study design}

This randomised, double-blind, placebo controlled, crossover study was approved by the Queen's University and Affiliated Hospitals research ethics board. After informed consent and screening of medical history, patients completed four visits conducted approximately 7 days apart. At visit 1, subjects completed pulmonary function tests and a symptom-limited incremental cycle exercise test followed, after $60 \mathrm{~min}$ of rest, by a familiarisation constant-load cycle endurance test at 80-85\% of their maximal achieved work rate (Wmax). At visit 2 the constant-load cycle test was repeated and, after $60 \mathrm{~min}$ of recovery, subjects performed pulmonary function tests before and $20 \mathrm{~min}$ after administration of salbutamol (400 $\mu \mathrm{g})$. At visits 3 and 4, subjects were randomised (sequence) to receive either nebulised ipratropium bromide $500 \mu \mathrm{g}$ (IB) or a placebo (PL). Subjects performed pulmonary function tests before and 60 min after nebulisation, followed by a constant-load exercise test. All series of pulmonary function tests included spirometry, body plethysmography, transfer factor and respiratory muscle strength measurements. All symptom-limited constant-load exercise tests were conducted at the same work rate for each subject. Withdrawal of bronchodilators before each visit included short-acting $\beta_{2}$ agonists $(8 \mathrm{~h})$, short-acting anticholinergics ( $8 \mathrm{~h})$, long-acting $\beta_{2}$ agonists (48 h) and longacting anticholinergics (72 h). Subjects avoided caffeine, alcohol and heavy meals for $4 \mathrm{~h}$ before visits and avoided major physical exertion entirely on visit days.

\section{Interventions}

A $3.5 \mathrm{ml}$ solution containing either $500 \mu \mathrm{g}$ IB or sterile $0.9 \%$ saline (PL) was administered by nebuliser (Parimaster Compressor with Pari LC Jet+nebuliser; PARI Respiratory Equipment, Richmond, Virginia, USA) over a 15-20 min period in a double-blind fashion.

\section{Procedures}

Routine spirometry, body plethysmography (ie, functional residual capacity (FRC) and specific airway resistance (sRaw)), transfer factor for carbon monoxide (TLCO) and maximum inspiratory and expiratory mouth pressures (MIP and MEP; measured at FRC and total lung capacity (TLC), respectively) were performed using an automated system (6200 Autobox DL or Vmax229d; SensorMedics, Yorba Linda, California, USA) in accordance with recommended techniques. ${ }^{11-16}$ Measurements were expressed as percentages of predicted normal values; ${ }^{17-19}$ predicted normal inspiratory capacity (IC) was calculated as predicted TLC minus predicted FRC.

Symptom-limited exercise tests were conducted on an electronically braked cycle ergometer as previously described. ${ }^{5021}$ The incremental test consisted of 2 min increments of $20 \mathrm{~W}$ to the point of symptom limitation; Wmax was defined as the greatest work rate that the subject could maintain for at least $30 \mathrm{~s}$. Constant-load tests at $80-85 \%$ Wmax were performed during all four visits; endurance time was defined as the duration of loaded pedalling. At end-exercise, subjects were asked why they needed to stop exercising. Rest was the steady-state period after at least 3 min of breathing on the mouthpiece before exercise began; peak was the last $30 \mathrm{~s}$ of loaded pedalling; and isotime was the duration of the shortest post-treatment test rounded down to the nearest full minute (ie, highest equivalent isotime).

Cardiopulmonary and breathing pattern measurements were collected in a breath-by-breath fashion while subjects breathed through a mouthpiece with nasal passages occluded by a noseclip using a cardiopulmonary exercise testing system (SensorMedics Vmax229d). Pulse oximetry, electrocardiography and blood pressure measurements were also performed. Subjects rated the intensity of their "breathing discomfort" and "leg discomfort" at rest, every minute during exercise and at endexercise using the modified 10-point Borg scale. ${ }^{22}$ Operating lung volumes were derived from IC measurements performed at rest, every second minute during exercise and end-exercise, as previously described. ${ }^{5}$ Maximal flow-volume loops were obtained at rest and at end-exercise. Tidal flow-volume curves at rest, every 2 min during exercise and at peak exercise were placed within their respective maximal flow-volume loops using coinciding IC measurements; expiratory flow limitation was estimated as the percentage of tidal volume (VT) encroaching on the maximal flow envelope. ${ }^{23}$ In six subjects, oesophageal pressure (Pes) was recorded continuously during constant-load exercise tests using an integrated data acquisition set-up as described elsewhere (see online supplement). ${ }^{20}$ Inspiratory sniff manoeuvres were performed before exercise at rest and immediately at end-exercise to obtain maximum values for Pes (PImax).

Table 1 Subject characteristics

\begin{tabular}{|c|c|c|}
\hline & $\begin{array}{l}\text { Enrolled subjects } \\
(\mathrm{n}=16)\end{array}$ & $\begin{array}{l}\text { Subjects with } \\
\text { complete } \\
\text { mechanical } \\
\text { measurements } \\
(n=6)\end{array}$ \\
\hline Gender & $63 \%$ male & $83 \%$ male \\
\hline Age (years) & $63(8)$ & $67(8)$ \\
\hline Body mass index $\left(\mathrm{kg} / \mathrm{m}^{2}\right)$ & $27.8(4.6)$ & $26.4(3.8)$ \\
\hline $\begin{array}{l}\text { Cigarette smoking history (pack- } \\
\text { years) }\end{array}$ & $44(16)$ & $43(18)$ \\
\hline BDI focal score (0-12) & $8.3(2.0)$ & $8.3(1.9)$ \\
\hline MRC dyspnoea scale (1-5) & $1.8(0.7)$ & $1.5(0.5)$ \\
\hline $\begin{array}{l}\text { CHAMPS (kcal/week consumed at } \\
\text { moderate activities) }\end{array}$ & $2123(2221)$ & $1736(1974)$ \\
\hline \multicolumn{3}{|l|}{$\begin{array}{l}\text { Symptom-limited peak exercise } \\
\text { (\% predicted maximum) }\end{array}$} \\
\hline Work rate $(\mathrm{W})$ & $121(39),(72 \%)$ & $119(30),(74 \%)$ \\
\hline$\dot{\mathrm{V}}_{2}(\mathrm{l} / \mathrm{min})$ & $1.84(0.58),(79 \%)$ & $1.79(0.58),(79 \%)$ \\
\hline \multicolumn{3}{|l|}{ Pulmonary function (\% predicted) } \\
\hline $\mathrm{FEV}_{1}$ post-bronchodilator (I) & $2.50(0.58),(90 \%)$ & $2.44(0.64),(86 \%)$ \\
\hline FVC post-bronchodilator (I) & 4.25 (1.08), (108\%) & 4.39 (1.18), (108\%) \\
\hline $\mathrm{FEV}_{1} / \mathrm{FVC}$ post-bronchodilator (\%) & $59(7),(84 \%)$ & $56(8),(80 \%)$ \\
\hline IC (I) & $3.01(0.97),(103 \%)$ & 3.34 (1.13), (106\%) \\
\hline FRC (I) & $4.10(0.91),(122 \%)$ & $4.45(0.71),(126 \%)$ \\
\hline TLC (I) & $7.11(1.50),(113 \%)$ & 7.79 (1.32), (117\%) \\
\hline RV (I) & $2.83(0.48),(129 \%)$ & $3.08(0.32),(131 \%)$ \\
\hline $\mathrm{MIP}\left(\mathrm{cm} \mathrm{H} \mathrm{H}_{2} \mathrm{O}\right)$ & $95(32),(115 \%)$ & $87(23),(95 \%)$ \\
\hline $\operatorname{MEP}\left(\mathrm{cm} \mathrm{H}_{2} \mathrm{O}\right)$ & 149 (59), (83\%) & 139 (44), (100\%) \\
\hline TLCo (ml/min/mm Hg) & $21.2(5.9),(95 \%)$ & $22.4(4.5),(106 \%)$ \\
\hline sRaw (cm H $\mathrm{H}_{2} \mathrm{O} . \mathrm{s}$ ) & $12.3(4.0),(294 \%)$ & $13.3(2.9),(303 \%)$ \\
\hline
\end{tabular}

Values are shown as mean (SD), (\% predicted normal values).

BDI, baseline dyspnoea index; MRC, Medical Research Council; CHAMPS, Community Healthy Activities Model Program for Seniors; $\mathrm{FEV}_{1}$, forced expiratory volume in $1 \mathrm{~s}$; FRC, functional reserve capacity; FVC, forced vital capacity; IC, inspiratory capacity; MIP, maximal inspiratory mouth pressure; MEP, maximal expiratory mouth pressure; RV, residual volume; sRaw, specific airway resistance; TLC, total lung capacity; TLCO, carbon monoxide transfer factor; $\dot{V}_{2}$, oxygen consumption. 
Table 2 Effect of placebo (PL) and ipratropium bromide (IB) on pulmonary function tests in patients with GOLD stage I COPD

\begin{tabular}{|c|c|c|}
\hline & Post-PL & Post-IB \\
\hline $\mathrm{FEV}_{1}$ (I) & $2.30(0.60),(83 \%)$ & $2.46(0.59),(88 \%)^{*}$ \\
\hline$\Delta \mathrm{FEV}_{1}(\mathrm{l})$ & $0.07(0.09),(3 \%) \dagger$ & $0.26(0.19),(9 \%) \uparrow \$$ \\
\hline FVC (I) & $4.07(1.13),(103 \%)$ & $4.20(1.17),(106 \%)^{*}$ \\
\hline$\Delta \mathrm{FVC}(\mathrm{l})$ & $0.02(0.18),(1 \%)$ & $0.23(0.21),(6 \%) \uparrow \$$ \\
\hline $\mathrm{FEV}_{1} / \mathrm{FVC}(\%)$ & 57 (7), (81\%) & $59(7),(83 \%)$ \\
\hline$\Delta \mathrm{FEV}_{1} / \mathrm{FVC}(\%)$ & $1.3(2.0),(2 \%) \dagger$ & $2.9(3.2),(4 \%) \dagger$ \\
\hline $\operatorname{TLCO}(\mathrm{ml} / \mathrm{min} / \mathrm{mm} \mathrm{Hg})$ & $21.0(6.2),(94 \%)$ & $20.2(6.5),(90 \%)$ \\
\hline$\Delta \mathrm{TLCO}(\mathrm{ml} / \mathrm{min} / \mathrm{mm} \mathrm{Hg})$ & $-0.1(1.4),(-1 \%)$ & $-1.1(2.3),(-5 \%)$ \\
\hline $\operatorname{MIP}\left(\mathrm{cm} \mathrm{H}_{2} \mathrm{O}\right)$ & $96(20),(120 \%)$ & 98 (21), (121\%) \\
\hline$\Delta \mathrm{MIP}\left(\mathrm{cm} \mathrm{H}_{2} \mathrm{O}\right)$ & $-1(11),(-2 \%)$ & $1.6(7.7),(1 \%)$ \\
\hline $\operatorname{MEP}\left(\mathrm{cm} \mathrm{H} \mathrm{H}_{2} \mathrm{O}\right)$ & 148 (54), (82\%) & $148(48),(83 \%)$ \\
\hline$\triangle \mathrm{MEP}\left(\mathrm{cm} \mathrm{H} \mathrm{H}_{2} \mathrm{O}\right)$ & $1(12),(1 \%)$ & $-6(13),(-10 \%)$ \\
\hline TLC (I) & $7.16(1.49),(114 \%)$ & 7.04 (1.42), (112\%) \\
\hline$\Delta \mathrm{TLC}$ (I) & $-0.03(0.26),(-1 \%)$ & $-0.15(0.20),(-3 \%) \dagger$ \\
\hline $\mathrm{RV}(\mathrm{I})$ & $2.73(0.34),(125 \%)$ & $2.49(0.47),(113 \%)^{*}$ \\
\hline$\Delta \mathrm{RV}(\mathrm{I})$ & $-0.14(0.19),(-6 \%) \dagger$ & $-0.38(0.22),(-19 \%) \dagger+$ \\
\hline FRC (I) & $4.05(0.71),(121 \%)$ & $3.90(0.82),(115 \%)^{*}$ \\
\hline$\Delta \mathrm{FRC}(\mathrm{I})$ & $-0.07(0.17),(-2 \%)$ & $-0.27(0.29),(-9 \%) \dagger$ \\
\hline IC (I) & $3.10(1.01),(106 \%)$ & $3.15(0.86),(109 \%)$ \\
\hline$\Delta \mathrm{IC}(\mathrm{l})$ & $0.04(0.19),(1 \%)$ & $0.12(0.32),(4 \%)$ \\
\hline sRaw (cm H ${ }_{2}$ O.s) & $12.7(4.3),(301 \%)$ & $9.3(4.6),(220 \%)^{*}$ \\
\hline$\Delta$ sRaw $\left(\mathrm{cm} \mathrm{H}_{2} 0 . \mathrm{s}\right)$ & $-0.6(1.7),(-15 \%)$ & $-4.7(3.5),(-111 \%) \dagger+$ \\
\hline
\end{tabular}

Values are mean (SD), (\% predicted normal values).

${ }^{*} p<0.05$, post-IB vs post-PL.

$\dagger p<0.05$ post-dose vs pre-dose within treatment.

$\$ p<0.05$ IB vs PL post-dose minus pre-dose differences.

$\Delta$, post-dose minus pre-dose difference; COPD, chronic obstructive pulmonary

disease; $\mathrm{FEV}_{1}$, forced expiratory volume in $1 \mathrm{~s}$; FVC, forced vital capacity;

IC, inspiratory capacity; MIP, maximal inspiratory pressure; MEP, maximal expiratory pressure; PEF, peak expiratory flow; RV, residual volume; sRaw, specific airway resistance; SVC, slow vital capacity; TLC, total lung capacity; TLCo, carbon monoxide transfer factor.

\section{Statistical analysis}

A sample size of 16 was used to provide the power (80\%) to detect a significant difference in dyspnoea intensity (Borg scale) measured at a standardised work rate during incremental cycle exercise based on a relevant difference in Borg ratings of \pm 1 , an $\mathrm{SD}$ of 1 for changes in Borg ratings found in our laboratory $(\alpha=0.05)$. Results were expressed as mean (SD). A $p<0.05$ level of statistical significance was used for all analyses.

Although unlikely in this single-dose study, the possibility of a carryover effect was tested using paired $t$ tests to evaluate predose (pre-treatment) pulmonary function measurements. Period effects were evaluated using the two-sample $t$ test. ${ }^{24}$ Treatment comparisons were made using paired $t$ tests with appropriate Bonferroni adjustments for multiple comparisons. Responses at rest and at different time points and/or intensities during exercise were also compared. Repeated measures ANOVA (with treatment, time and interaction as fixed effects and subject as a random effect) was applied to compare the overall treatment effects. Dyspnoea descriptors were analysed as frequency statistics and compared using the Fisher exact test. Physiological contributors to exertional dyspnoea intensity were determined by multiple regression analysis: Borg dyspnoea ratings at a standardised exercise work rate (dependent variable) were analysed against concurrent relevant independent variables (ie, exercise measurements of ventilation, breathing pattern, operating lung volumes, cardiovascular and metabolic parameters, and baseline pulmonary function measurements).
Table 3 Post-dose peak of symptom-limited constant-load exercise at 80-85\% Wmax (99 (32) W)

\begin{tabular}{|c|c|c|}
\hline & Placebo & Ipratropium bromide \\
\hline Exercise time (min) & $8.2(5.3)$ & $8.2(4.8)$ \\
\hline Dyspnoea (Borg scale) & $7.8(2.9)$ & $7.7(2.5)$ \\
\hline Leg discomfort (Borg scale) & $8.6(1.9)$ & $8.4(2.2)$ \\
\hline \multicolumn{3}{|l|}{ Reason for stopping, $\mathrm{n}(\%)$ : } \\
\hline Breathing & $3(19 \%)$ & $2(13 \%)$ \\
\hline Legs & $6(37 \%)$ & $9(56 \%)$ \\
\hline Breathing and legs & $7(44 \%)$ & $5(31 \%)$ \\
\hline$\dot{\mathrm{V}}_{2}(\mathrm{l} / \mathrm{min})$ & $1.88(0.64)$ & $1.81(0.56)$ \\
\hline$\dot{\mathrm{V}} \mathrm{CO}_{2}(\mathrm{l} / \mathrm{min})$ & $1.73(0.55)$ & $1.75(0.58)$ \\
\hline$\dot{V}_{E}(\mathrm{l} / \mathrm{min})$ & $69.4(18.2)$ & $73.5(23.9)$ \\
\hline$F$ (breaths/min) & $39.6(7.9)$ & $39.1(7.3)$ \\
\hline VT (I) & $1.81(0.56)$ & $1.91(0.58)^{*}$ \\
\hline IC (I) & $2.46(0.70)$ & $2.59(0.70)$ \\
\hline IRV (I) & $0.65(0.26)$ & $0.67(0.32)$ \\
\hline $\mathrm{VT} / \mathrm{TE}_{\mathrm{E}}(\mathrm{l} / \mathrm{s})$ & $2.10(0.59)$ & $2.24(0.80)$ \\
\hline $\mathrm{VT} / \mathrm{TI}(\mathrm{l} / \mathrm{s})$ & $2.56(0.63)$ & $2.68(0.80)$ \\
\hline Ті/Ттот & $0.45(0.03)$ & $0.45(0.03)$ \\
\hline $\mathrm{PetCO}_{2}(\mathrm{~mm} \mathrm{Hg})$ & $34.4(4.7)$ & $33.0(5.4)$ \\
\hline Heart rate (beats/min) & $142(16)$ & $140(17)$ \\
\hline $\mathrm{SpO}_{2}(\%)$ & $95(3)$ & $95(2)$ \\
\hline
\end{tabular}

Values are mean (SD)

${ }^{*} p<0.05$ ipratropium bromide versus placebo.

IC, inspiratory capacity; IRV, inspiratory reserve volume; $\mathrm{PETCO}_{2}$, partial pressure of end-tidal $\mathrm{CO}_{2} ; \mathrm{SpO}_{2}$, oxygen saturation; $\mathrm{T} / / \mathrm{TTOT}$, inspiratory duty cycle, inspiratory time over total breath time; $\mathrm{V}_{2}$, oxygen uptake; $\dot{V}_{\mathrm{CO}_{2}}$, carbon dioxide production;

$\dot{\mathrm{V}}_{\mathrm{E}}$, minute ventilation; $F$, breathing frequency; $\mathrm{V}_{\mathrm{T}}$, tidal volume; $\mathrm{VT} / \mathrm{TE}$ and $\mathrm{VT} / \mathrm{T}$, mean inspiratory and expiratory tidal flows.

\section{RESULTS}

\section{Subjects}

Subject characteristics are summarised in table 1. All subjects were had symptoms and had a diagnosis of COPD; the majority $(11 / 16)$ had a diagnosis made within the previous 5 years. Seven subjects did not use any respiratory medications, two only used a short-acting $\beta_{2}$ agonist bronchodilator on an "as needed" basis and seven used inhalers on a regular basis. Of these latter seven subjects, all used short-acting $\beta_{2}$ agonists, five used a long-acting $\beta_{2}$ agonist, five used an anticholinergic (one short-acting, four long-acting) and six used an inhaled corticosteroid (five in combination with a long-acting $\beta_{2}$ agonist). Comorbidities included stable coronary artery disease $(n=2)$, well-controlled diabetes mellitus type $2(n=1)$, treated hypertension $(n=1)$ and varying degrees of osteoarthritis $(n=4)$. All subjects had a smoking history of $\geqslant 15$ pack-years (range 15-63 pack-years, table 1); four subjects were current smokers and 12 were exsmokers who had stopped smoking at least 2 years before the study.

Chronic activity-related dyspnoea was assessed with the baseline dyspnoea index (BDI $)^{25}$ and the Medical Research Council (MRC) dyspnoea scale. ${ }^{26} \mathrm{BDI}$ focal scores ranged from 5 to 12; nine subjects reported a $\mathrm{BDI} \leqslant 8$ and seven subjects reported a $\mathrm{BDI} \geqslant 9$. The majority of subjects $(11 / 16)$ had a rating of $\geqslant 2$ on the MRC dyspnoea scale.

All subjects had a normal post-bronchodilator $\mathrm{FEV}_{1}$ and an $\mathrm{FEV}_{1} / \mathrm{FVC}$ ratio $<70 \%$. Lung volumes indicated mild static lung hyperinflation (mean FRC and RV $>120 \%$ predicted) with a preserved vital capacity and inspiratory capacity (IC) (table 1). Symptom-limited incremental exercise testing showed reduced peak oxygen consumption $\left(\mathrm{V}_{2}\right)$ and work rate. The subgroup of subjects with Pes-derived measurements had comparable baseline characteristics to the group as a whole (table 1). There 
were also no significant differences in the baseline characteristics or in the magnitude of the treatment responses between patients who were using respiratory medication and those who were not.

There were no significant differences between predose measurements of pulmonary function on treatment days (ie, no significant carryover effect). No significant period effects were found when examining pulmonary function or exercise test outcomes.

\section{Pulmonary function responses}

Differences in pulmonary function after IB compared with PL are shown in table 2. Subjects with the worst baseline prebronchodilator specific airway resistance (sRaw) had the greatest IB-induced improvements in sRaw $(r=-0.595$, $\mathrm{p}=0.015)$ and IC $(\mathrm{r}=0.594, \mathrm{p}=0.015)$; improvements in sRaw and IC were also strongly interrelated $(r=-0.680$, $p=0.004)$. Maximal expiratory flows measured at FRC after PL were compared with the maximal flow at the same absolute volume after IB within each individual: these isovolume flows improved from a mean (SD) of $0.27(0.18) \mathrm{l} / \mathrm{s}$ to $0.43(0.30) \mathrm{l} / \mathrm{s}$ after PL and IB, respectively $(p=0.006)$.

\section{Responses to constant-load exercise}

Post-dose exercise endurance time at 99 (32) W (60 (11)\% predicted maximum; 82 (9)\% Wmax) did not change significantly after IB compared with $\mathrm{PL}$ (table 3): six subjects improved endurance time by $\geqslant 30 \mathrm{~s}$, three subjects decreased endurance time by $\geqslant 30 \mathrm{~s}$ and the rest had less than a $30 \mathrm{~s}$ difference in endurance time. The distribution of reasons for stopping exercise was not different between treatments. In both visits, leg discomfort was reported as the primary reason for stopping exercise, 2-3-fold more than breathing discomfort (table 3).

\section{Exertional symptoms}

Compared with PL, there was no change in peak Borg ratings of breathing or leg discomfort after IB (table 3 ). However, ratings of breathing and leg discomfort at the highest equivalent isotime (6.8 (4.5) $\mathrm{min})$ during exercise were lower after IB compared with PL by 0.88 (1.83) Borg units $(p=0.073)$ and 0.81 (1.28) Borg units $(p<0.05)$, respectively (table 4 , fig 1$)$. Ten of the 16 patients decreased the intensity of their breathing discomfort at isotime by at least 1 Borg unit, while the remaining subjects increased $(n=4)$ or did not change $(n=2)$
Table 4 Post-dose values at isotime $(6.8$ (4.5) $\mathrm{min})$ during constantload exercise

\begin{tabular}{|c|c|c|c|}
\hline & Placebo & $\begin{array}{l}\text { Ipratropium } \\
\text { bromide }\end{array}$ & p Value \\
\hline Dyspnoea (Borg) & $7.4(2.4)$ & $6.6(2.4)$ & 0.07 \\
\hline Dyspnoea/VE (Borg///min) & $0.12(0.05)$ & $0.10(0.05)^{*}$ & \\
\hline Leg discomfort (Borg) & $8.3(1.7)$ & $7.5(2.1)^{*}$ & \\
\hline$\dot{\mathrm{V}}_{2}(\mathrm{l} / \mathrm{min})$ & $1.72(0.54)$ & $1.82(0.66)$ & \\
\hline$\dot{\mathrm{V}} \mathrm{co}_{2}(\mathrm{l} / \mathrm{min})$ & $1.67(0.56)$ & $1.71(0.62)$ & \\
\hline Heart rate (beats/min) & $137(19)$ & $136(19)$ & \\
\hline $\mathrm{SpO}_{2}(\%)$ & $96(3)$ & $96(2)$ & \\
\hline$\dot{\mathrm{V}}_{\mathrm{E}}(\mathrm{l} / \mathrm{min})$ & $65.7(19.6)$ & $69.0(24.9)$ & \\
\hline$F$ (breaths/min) & $36.7(8.3)$ & $35.3(8.2)$ & \\
\hline VT (I) & $1.83(0.57)$ & $1.99(0.65)^{*}$ & \\
\hline IC (I) & $2.51(0.70)$ & $2.63(0.66)^{*}$ & \\
\hline$\Delta \mathrm{IC}$ isotime-rest (I) & $-0.55(0.40)$ & $-0.49(0.33)$ & \\
\hline IRV (I) & $0.68(0.31)$ & $0.64(0.30)$ & \\
\hline $\mathrm{Tl}(\mathrm{s})$ & $0.77(0.20)$ & $0.81(0.21)$ & \\
\hline $\mathrm{TE}(\mathrm{s})$ & $0.93(0.20)$ & $0.98(0.23)$ & 0.06 \\
\hline ТІ/Ттот & $0.45(0.03)$ & $0.45(0.03)$ & \\
\hline VD/VT estimated (\%) & $30(7)$ & $29(8)^{*}$ & \\
\hline $\mathrm{PeTCO}_{2}(\mathrm{~mm} \mathrm{Hg})$ & $35.4(5.1)$ & $34.3(5.5)$ & \\
\hline $\begin{array}{l}\text { EFL (\% of VT overlapping maximal } \\
\text { flow-volume curve) }\end{array}$ & $78(13)$ & $62(20)^{*}$ & \\
\hline
\end{tabular}

Values are mean (SD).

${ }^{*} p<0.05$ ipratropium bromide vs placebo.

$E F L$, expiratory flow limitation; IC, inspiratory capacity; IRV, inspiratory reserve volume; $\mathrm{PETCO}_{2}$, partial pressure of end-tidal $\mathrm{CO}_{2} ; \mathrm{SpO}_{2}$, oxygen saturation; $\mathrm{TI} / \mathrm{TTOT}$, inspiratory duty cycle, inspiratory time over total breath time; $\dot{V}_{2}$, oxygen uptake; $\dot{V} \mathrm{CO}_{2}$, carbon dioxide production; $\dot{V}_{\mathrm{E}}$, minute ventilation; $F$, breathing frequency; VC, vital capacity; $V_{D}$, estimated dead space; $V_{T}$, tidal volume.

ratings of breathing discomfort after IB compared with PL. Dyspnoea/VE ratios were evaluated to account for the potential effects of IB-induced alterations in $\mathrm{VE}$ on exertional dyspnoea intensity: dyspnoea/VE ratios were significantly lower $(p<0.05)$ at isotime after IB than after PL (table 4, fig 1). By repeated measures ANOVA, there were no significant interactions between treatment and time during exercise for exertional symptom ratings (ie, the treatment effect did not vary at different times); however, a significant treatment effect was found for dyspnoea $/ \mathrm{VE}$ ratios $(p=0.075)$ and leg discomfort ratings $(p=0.021)$.

\section{Ventilatory responses}

Ventilatory responses to exercise after IB and PL are shown in fig 2. Tidal volume (VT) was greater after IB compared with PL
Figure 1 Ratings of dyspnoea intensity, expressed relative to ventilation, and ratings of intensity of leg discomfort plotted against exercise time during constant-load cycle testing at $80-85 \%$ of the maximum work rate achieved during incremental testing. ${ }^{*} p<0.05$ ipratropium bromide vs placebo at a given time point. Values are mean (SEM).
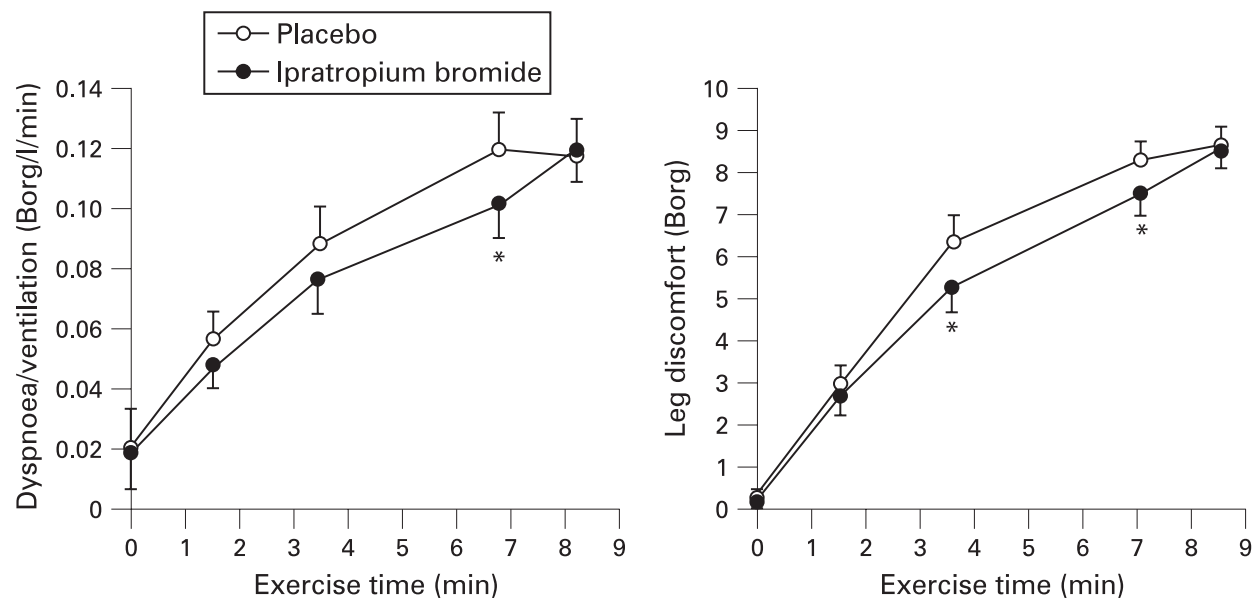
Figure 2 Ventilatory responses to constant-load cycle testing against exercise time after ipratropium bromide (IB) compared with placebo (PL) in 16 subjects with mild chronic obstructive pulmonary disease. Tidal volume (VT) and inspiratory capacity were greater, and estimates of expiratory flow limitation (EFL) were lower after IB than after PL; minute ventilation, breathing frequency $(F)$ and inspiratory reserve volume were not different between treatments.

TLC, total lung capacity. ${ }^{*} \mathrm{p}<0.05$ ipratropium bromide vs placebo at a given time point or at peak exercise. Values are mean (SEM).
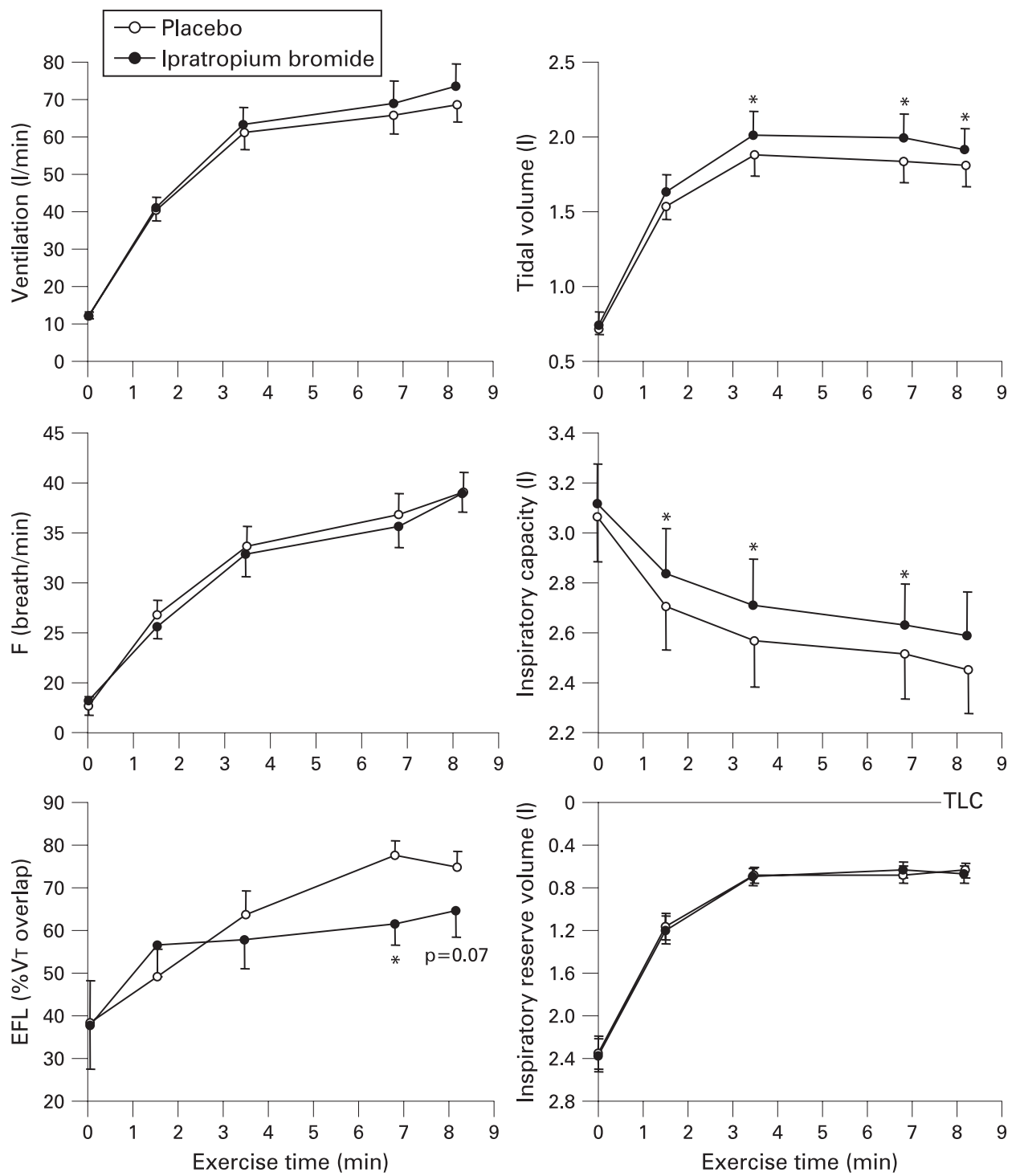

from minute 4 in exercise to peak exercise by $0.10-0.161$ $(p<0.05)$; increases in VT were accommodated by concurrent increases in IC of $0.12-0.151 \quad(p<0.05)$. Inspiratory reserve volume (IRV) was not different at rest or throughout exercise across treatments. Estimates of expiratory flow limitation were reduced at isotime and at peak exercise by $10 \%$ and $17 \%$ $(p<0.05)$, respectively, after IB compared with PL. Repeated measures ANOVA also showed a significant treatment effect for VT $(p=0.012)$, IC $(p=0.001)$ and expiratory flow limitation $(p=0.014)$, with no significant interactions between treatment and exercise time.

\section{Ventilatory mechanics}

The pressure-time integral and its surrogate, the calculated tension-time index (Pes/PImax $\times$ TI/TTOT), were not different in response to treatment. However, lung resistance was reduced after IB compared with PL in the order of $0.7-0.8 \mathrm{~cm} \mathrm{H}_{2} \mathrm{O} / \mathrm{l} / \mathrm{s}$ at standardised time points throughout exercise (ie, a reduction of $\sim 20 \%$ ( $p<0.05$ ), fig 3). Total work of breathing expressed as J/1 fell significantly $(p<0.05)$ during exercise but not at rest after IB compared with PL, primarily due to significant $(p<0.05)$ decreases in the inspiratory threshold load and the elastic work performed against this load. Repeated measures ANOVA showed a significant treatment effect for resistance $(p=0.007)$, the inspiratory threshold load $(p<0.001)$, total work of breathing $(p=0.002)$ and elastic work of breathing $(p=0.001)$, with no significant interactions between treatment and exercise time. Work of breathing measurements were not different when expressed as J/min, so differences were offset by increases in $\mathrm{VE}$.

\section{Correlates of dyspnoea}

The best predictors of the IB-induced decrease in dyspnoea ratings at isotime were the baseline (prebronchodilator) preexercise resting IC expressed as percentage predicted $(r=0.637$, $p=0.008)$, the end-inspiratory lung volume (EILV)/TLC ratio $(\mathrm{r}=-0.561, \mathrm{p}=0.024)$ and the IRV expressed as percentage predicted TLC $(r=541, p=0.030)$; no other baseline pulmonary function parameters correlated. The best correlates of the IBinduced decrease in dyspnoea/VE ratios at isotime were also the prebronchodilator pre-exercise resting IC expressed as percentage predicted $(r=0.714, p=0.002)$, the EILV/TLC ratio $(\mathrm{r}=-0.637, \mathrm{p}=0.008)$ and the IRV expressed as percentage predicted TLC $(r=0.549, p=0.028)$. When dyspnoea was 
Figure 3 Respiratory mechanical measurements in six subjects during constant-load exercise after ipratropium bromide compared with placebo. Pulmonary resistance, inspiratory threshold load, total work of breathing and the elastic work of breathing component all decreased during exercise after ipratropium bromide compared with placebo. However, peak tidal inspiratory and expiratory oesophageal pressure (Pes) did not change in response to treatment. ${ }^{*} \mathrm{p}<0.05$ (one-tailed) ipratropium bromide vs placebo at a given time point or at peak exercise. Values are mean (SEM).
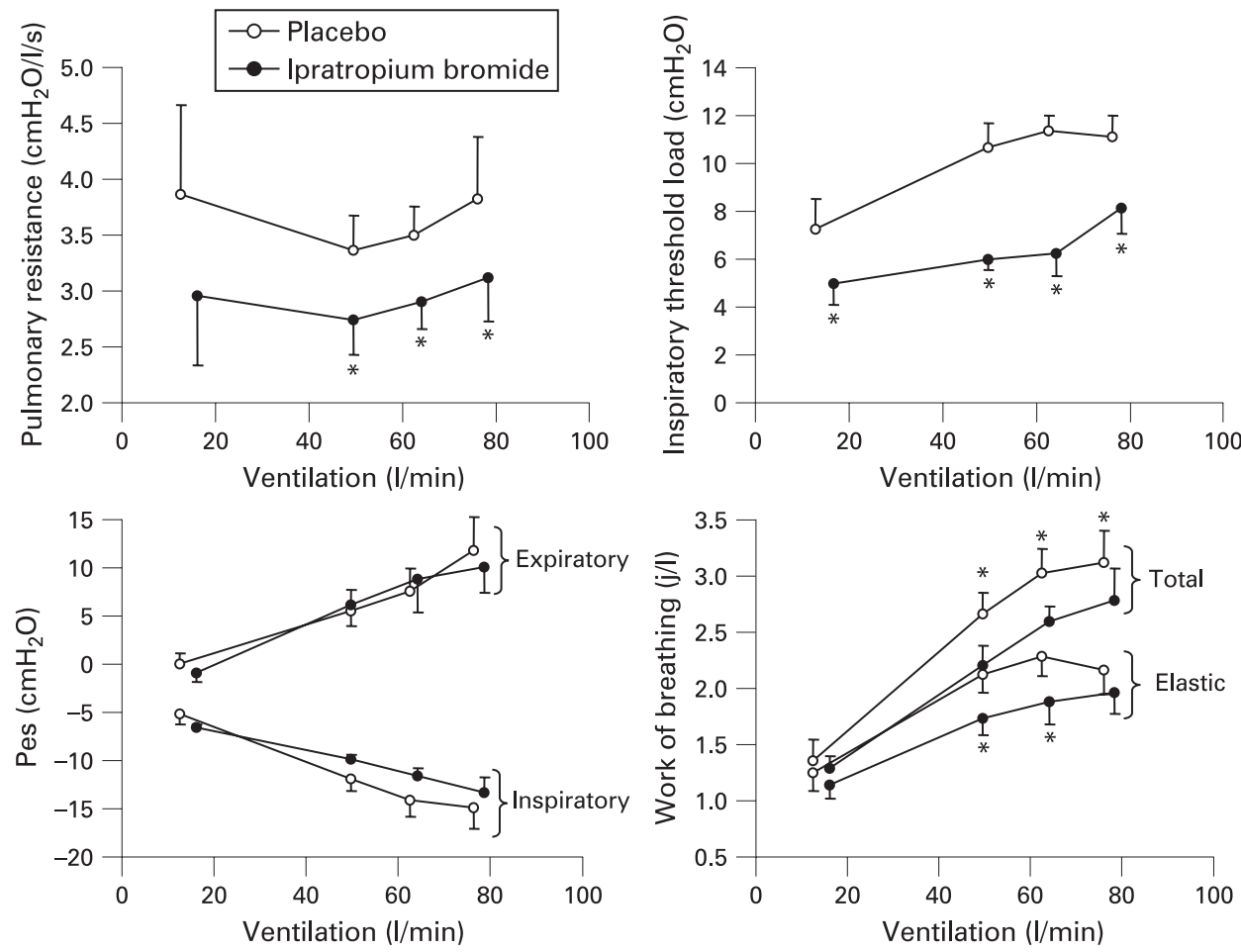

expressed as a ratio against $\operatorname{VE}$ (Borg/(1/min)), the strongest correlate of this treatment difference at isotime was the concurrent difference in EELV/TLC $(r=0.585, p<0.05)$.

\section{DISCUSSION}

The novel findings of this study are as follows: (1) treatment with IB was associated with consistent improvements in forced expiratory flow rates, sRaw and RV in patients with GOLD stage I COPD symptoms; (2) during exercise, IB treatment was associated with significant increases in dynamic IC and VT in the absence of an increase in cycle exercise endurance time; and (3) improvement in dynamic EELV was linked to a reduction in ratings of exertional dyspnoea intensity at higher levels of ventilation.

\section{Changes in resting pulmonary function}

Our patients had extensive physiological impairment and longterm activity-related dyspnoea as measured by validated questionnaires. More than half of the group was already receiving empirical bronchodilator therapy. Our results confirm that release of cholinergic smooth muscle tone improved airway function both at rest and during exercise in these patients. In general, changes in resting spirometry and lung volumes were in the same direction but more modest than those previously reported following a similar dose of IB in moderate to severe COPD. ${ }^{7-9}$ Airway resistance, corrected for the lower resting operating volume, decreased after IB by $33 \%$ of baseline values while isovolume maximal flow rates in the effort-independent range also consistently improved.

In contrast to previous studies of the acute effects of IB in more advanced disease, resting IC did not increase significantly in our cohort with milder COPD. Thus, FRC and TLC fell in tandem and to a similar extent, such that the change in IC underestimated the extent of IB-induced lung deflation. Small but consistent bronchodilator-associated decreases in TLC have previously been reported in COPD, but the precise mechanisms are unknown. ${ }^{27}{ }^{28}$ Decreases in the plethysmographically-determined TLC may reflect measurement artefact, since mouth pressure during panting could potentially underestimate true alveolar pressure in patients with airflow limitation. A reduction in this disparity after a bronchodilator may result in an artifactual reduction in TLC. We tried to minimise this effect by controlling panting frequency at $\sim 1 \mathrm{~Hz}$. It is unlikely that changes in (regional) lung compliance can explain the observed reductions in TLC; lung compliance curves were superimposed before and after IB in our small subsample with mechanical measurements.

The lack of increase in resting IC in our patients is not surprising. Based on the study of Tantucci et al, ${ }^{29}$ bronchodilator-induced increases in IC are only expected in patients with COPD who have more extensive expiratory flow limitation and lung hyperinflation (ie, IC $<80 \%$ predicted) at rest.

\section{Altered ventilatory responses to exercise after bronchodilator} Exercise endurance time did not increase after bronchodilator compared with placebo. Possible explanations for this are: (1) the study was powered to detect an improvement in dyspnoea at a standardised work rate and not a change in exercise endurance time; and (2) intolerable leg discomfort and not dyspnoea was the dominant exercise-limiting symptom in the majority of this group.

IC diminished by 0.551 from rest to peak exercise, confirming the presence of air trapping due to expiratory flow limitation and high ventilatory demand in patients with mild COPD. Compared with placebo, IB treatment was associated with a significant increase in IC by $0.12-0.151$ throughout exercise, despite slightly greater levels of ventilation $(\sim 3 \mathrm{l} / \mathrm{min})$. However, the magnitude of acute change in IC from preexercise resting levels at each time point and at peak exercise remained similar (fig 2 ). We also found consistent reductions in our estimate of expiratory flow limitation at higher exercise levels after IB. Moreover, pulmonary resistance and 
conductance were significantly improved during exercise in the subsample who consented to oesophageal balloon measurements. The most likely explanation for lung deflation is therefore improvement in the time constant for lung emptying as a result of reduced airway resistance rather than minimal changes in expiratory time (prolongation) and static lung recoil pressure. The improved dynamic IC allowed greater VT expansion throughout exercise without further encroachment on the dynamic IRV.

\section{Mechanisms of dyspnoea relief}

Standardised ratings of exertional dyspnoea intensity as measured by the Borg scale were not statistically different $(p=0.07)$ after IB compared with placebo, probably due to small concomitant increases in VE with IB. However, when IBinduced alterations in VE were taken into account by examining dyspnoea/VE ratios, these changes reached statistical significance $(p<0.05)$. Compared with placebo, the decrease in the dyspnoea/VE ratio after IB correlated best with the concurrent decrease in the dynamic EELV/TLC ratio. In a small subset of patients the measured work of breathing and the pressure-time product (reflecting the oxygen cost of breathing) was not increased after IB despite significantly greater VT expansion. The work associated with overcoming the inspiratory threshold load (the intrinsic PEEP effect) was significantly reduced at standardised times during exercise. We have argued that increased threshold loading of the inspiratory muscles as a result of dynamic pulmonary hyperinflation plays an important role in dyspnoea causation in asthma during bronchoconstriction and in more advanced COPD during exercise. ${ }^{30-32}$ Reduction of the inspiratory threshold load by lung deflation should relieve dyspnoea by reducing the disparity between efferent motor output (sensed by increased corollary discharge) and afferent inputs from mechanosensors in the respiratory muscles, chest wall and lungs (ie, neuromechanical coupling). ${ }^{33}$ Thus, dyspnoea relief was related to improved inspiratory muscle function as a result of a reduced dynamic EELV as well as the recruitment of an increased dynamic IC which allowed greater VT displacement for the same inspiratory effort.

It is noteworthy that patients who derived the greatest reduction in exertional dyspnoea with IB treatment were those with the most severe lung hyperinflation at baseline. In fact, of all the resting physiological parameters that we measured, only the resting prebronchodilator IC and IRV (percentage predicted) correlated with improved dyspnoea intensity ratings during exercise. Previous studies have shown that, when the normal spontaneous VT response to increasing central respiratory drive is constrained (either volitionally or by imposition), dyspnoea quickly escalates to intolerable levels. ${ }^{34}{ }^{35}$ It follows that release of VT restriction (ie, IC recruitment) should improve dyspnoea. ${ }^{21} 36$

In summary, traditional spirometric measurements reliably detected modest but consistent improvements in airway function after bronchodilator treatment in patients with symptoms of mild COPD. Bronchodilator administration was associated with improved dynamic IC and a deeper breathing pattern throughout exercise. Dyspnoea intensity ratings fell only at the higher levels of ventilation with IB treatment, in association with reduced dynamic EELV. Mechanical and subjective improvements during exercise after IB treatment were most pronounced in those with the smallest resting IC (and IRV) and therefore the greatest mechanical constraints on tidal volume expansion.
This study highlights the challenges involved in the assessment of bronchodilator efficacy in milder COPD where no evidence-based guidelines for pharmacotherapy currently exist. Our results provide a sound physiological rationale for consideration of a trial of bronchodilator therapy in selected patients with GOLD stage I COPD who experience troublesome activity-related dyspnoea.

Funding: Supported by William M Spear endowment fund, Queen's University. Competing interests: None.

Ethics approval: This study was approved by the Queen's University and Affiliated Hospitals research ethics board.

\section{REFERENCES}

1. Buist AS, Ross BB. Quantitative analysis of the alveolar plateau in the diagnosis of early airway obstruction. Am Rev Respir Dis 1973;108:1078-87.

2. Anthonisen NR, Connett JE, Murray RP. Smoking and lung function of Lung Health Study participants after 11 years. Am J Respir Crit Care Med 2002;166:675-9.

3. Hogg JC, Chu F, Utokaparch S, et al. The nature of small-airway obstruction in chronic obstructive pulmonary disease. N Engl J Med 2004;350:2645-53.

4. Verbanck S, Schuermans D, Paiva M, et al. Small airway function improvement after smoking cessation in smokers without airway obstruction. Am J Respir Crit Care Med 2006;174:853-7.

5. Ofir D, Laveneziana P, Webb KA, et al. Mechanisms of dyspnea during cycle exercise in symptomatic patients with GOLD stage I COPD. Am J Respir Crit Care Med 2008;177:622-9.

6. Jones PW. Health status measurement in chronic obstructive pulmonary disease. Thorax 2001;56:880-7.

7. O'Donnell DE, Lam M, Webb KA. Spirometric correlates of improvement in exercise performance after anticholinergic therapy in chronic obstructive pulmonary disease. Am J Respir Crit Care Med 1999;160:553-9.

8. Ayers ML, Mejia R, Ward J, et al. Effectiveness of salmeterol versus ipratropium bromide on exertional dyspnoea in COPD Eur Respir J 2001;17:1132-7.

9. Oga T, Nishimura K, Tsukino M, et al. The comparison of the effects of salbutamol and ipratropium bromide on exercise endurance in patients with COPD. Chest 2003;123:1810-6.

10. Rabe KF, Hurd S, Anzueto A, et al. Global strategy for diagnosis, management, and prevention of chronic obstructive pulmonary disease: GOLD executive summary. Am J Respir Crit Care Med 2007;176:532-55.

11. American Thoracic Society, European Respiratory Society. ATS/ERS statement on respiratory muscle testing. Am J Respir Crit Care Med 2002;166:518-624.

12. MacIntyre N, Crapo RO, Viegi G, et al. Standardisation of the single-breath determination of carbon monoxide uptake in the lung. Eur Respir J 2005;26:720-35.

13. Miller MR, Crapo R, Hankinson J, et al on behalf of the ATS/ERS Task Force. General considerations for lung function testing. Eur Respir J 2005;26:153-61.

14. Miller MR, Hankinson J, Brusasco V, et al. Standardisation of spirometry. Eur Respir J 2005;26:319-38.

15. Wanger J, Clausen JL, Coates A, et al. Standardisation of the measurement of lung volumes. Eur Respir J 2005;26:511-22.

16. Blonshine S, Foss C, Ruppel G, Wanger J; American Association for Respiratory Care. AARC clinical practice guideline: Body plethysmography - 2001 revision and update. Respir Care 2001;46:506-13.

17. Burrows B, Kasik JE, Niden AH, et al. Clinical usefulness of the single-breath pulmonary diffusing capacity test. Am Rev Respir Dis 1961;84:789-806.

18. Crapo RO, Morris AH, Clayton PD, et al. Lung volumes in healthy nonsmoking adults Bull Eur Physiopathol Respir 1982;18:419-25.

19. Morris JF, Koski A, Temple WP, et al. Fifteen-year interval spirometric evaluation of the Oregon predictive equations. Chest 1988;93:123-7.

20. O'Donnell DE, Hamilton AL, Webb KA. Sensory-mechanical relationships during high intensity, constant work rate exercise in COPD. J Appl Physiol 2006;101:1025-35.

21. Ofir D, Laveneziana $P$, Webb KA, et al. Ventilatory and perceptual responses to cycle exercise in obese women. J Appl Physiol 2007;102:2217-26.

22. Borg GA. Psychophysical basis of perceived exertion. Med Sci Sports Exerc 1982;14:377-81.

23. Johnson BD, Weisman IM, Zeballos RJ, et al. Emerging concepts in the evaluation of ventilatory limitation during exercise: the exercise tidal flow-volume loop. Chest 1999:116:488-503.

24. Senn S. Cross-over trials in clinical research. 2nd ed. Etobicoke, Ontario, Canada: John Wiley, 2002:35-88.

25. Mahler DA, Weinberg DH, Wells CK, et al. The measurement of dyspnea. Contents, interobserver agreement, and physiologic correlates of two new clinical indexes. Chest 1984:85:751-8.

26. Fletcher CM, Elmes PC, Fairbairn AS, et al. The significance of respiratory symptoms and the diagnosis of chronic bronchitis in a working population. BMJ 1959;2:257-66

27. O'Donnell DE, Forkert L, Webb KA. Evaluation of bronchodilator responses in patients with "irreversible" emphysema. Eur Respir J 2001;18:914-20.

28. O'Donnell D, Flüge T, Gerken F, et al. Effects of tiotropium on lung hyperinflation, dyspnoea and exercise tolerance in COPD. Eur Respir J 2004;23:832-40. 
29. Tantucci C, Duguet A, Similowski T, et al. Effect of salbutamol on dynamic hyperinflation in chronic obstructive pulmonary disease patients. Eur Respir J 1998;12:799-804.

30. Lougheed MD, Webb KA, O'Donnell DE. Breathlessness during induced hyperinflation in asthma: the role of the inspiratory threshold load. Am J Respir Crit Care Med 1995;152:911-20

31. O'Donnell DE, Sanii R, Giesbrecht G, et al. Effect of continuous positive airway pressure on respiratory sensation in patients with chronic obstructive pulmonary disease during submaximal exercise. Am Rev Respir Dis 1988;138:1185-91.

32. O'Donnell DE, Bertley JC, Chau LKL, et al. Qualitative aspects of exertional breathlessness in chronic air limitation. Am J Respir Crit Care Med 1997;155:109-15.
33. O'Donnell DE, Banzett RB, Carrieri-Kohlman V, et al. Pathophysiology of dyspnea in chronic obstructive pulmonary disease: a roundtable. Proc Am Thorac Soc 2007:4:145-68

34. Manning HL, Shea SA, Schwartzstein RM, et al. Reduced tidal volume increases "air hunger" at fixed $\mathrm{PCO}_{2}$ in ventilated quadriplegics. Respir Phys 1992;90:19-30

35. O'Donnell DE, Hong HH, Webb KA. Respiratory sensation during chest wall restriction and dead space loading in exercising men. J Appl Physiol 2000;88:1859-69.

36. O'Donnell DE, Voduc N, Fitzpatrick M, et al. Effect of salmeterol on the ventilatory response to exercise in chronic obstructive pulmonary disease. Eur Respir $J$ 2004;24:86-94. 